\title{
Polarimetric PALSAR System Model Assessment and Calibration
}

\author{
PI Number 228 \\ R. Touzi ${ }^{1}$ and M. Shimada ${ }^{2}$ \\ ${ }^{1}$ Canada Centre for Remote Sensing $\quad{ }^{2}$ Japan Aerospace exploration agency \\ Natural Resources Canada \\ 588 Booth Street, \\ Ottawa, Ontario, Canada K1A 0Y7 \\ Sengen 2-1-1 \\ Tsukuba, Ibaraki \\ Japan, 305-8505
}

\begin{abstract}
Polarimetric PALSAR system parameters are assessed using data sets collected over various calibration sites. The data collected over the Amazonian forest permits validating the zero Faraday rotation hypotheses near the equator. The analysis of the Amazonian forest data and the response of the corner reflectors deployed during the PALSAR acquisitions lead to the conclusion that the antenna is highly isolated (better than $-35 \mathrm{~dB}$ ). Theses results are confirmed using data collected over the Sweden and Ottawa calibration sites. The 5-m height trihedrals deployed in the Sweden calibration site by the Chalmers University of technology permits accurate measurement of antenna parameters, and detection of 2-3 degree Faraday rotation during day acquisition, whereas no Faraday rotation was noted during night acquisition. Small Faraday rotation angles (2-3 degree) have been measured using acquisitions over the DLR Oberpfaffenhofen and the Ottawa calibration sites. The presence of small but still significant Faraday rotation (2-3 degree) induces a CR return at the crosspolarization $\mathrm{HV}$ and $\mathrm{VH}$ that should not be interpreted as the actual antenna cross-talk. PALSAR antenna is highly isolated (better than $-35 \mathrm{~dB}$ ), and diagonal antenna distortion matrices (with zero cross-talk terms) can be used for accurate calibration of PALSAR polarimetric data.

KEYWORDS: Radar Polarimetry, Synthetic aperture radar, Calibration, Faraday rotation.
\end{abstract}

\section{INTRODUCTION}

PALSAR, L-band SAR onboard ALOS [1], is equipped with an active phased array $\mathrm{H}-\mathrm{V}$ antenna and two receivers that permit an alternating measurement of the backscattered wave at $\mathrm{HH}, \mathrm{HV}$ and $\mathrm{VV}, \mathrm{VH}$ polarizations [1], [2]. Like L-band JERS-1 SAR [3], ALOS PALSAR should be affected by Faraday rotation. The eventual presence of Faraday rotation in addition to the uncertainty regarding the actual isolation of the PALSAR antenna, lead to mixed conclusions regarding the actual isolation of the H-V PALSAR antenna [4], [5]. In the last Pol-In SAR07 workshop, it was concluded that the antenna isolation is not better than -25 dB (http : //earth.esa.int/workshops/polinsar 2007). Such a low isolation should make the system calibration complicate in the presence of Faraday rotation and mainly for areas with significant topographic relief.

In this paper, ALOS-PALSAR data sets collected over various calibration sites are used for the assessment and validation of the polarimetric PALSAR system parameters. In each site, one or several corner reflectors (CR) have been deployed during the ALOS acquisition for calibration purposes. In this study, we will use data sets col- lected over the Amazonian forest, the CCRS-Ottawa calibration site, as well as the calibration sites of DLR [4] and the Chalmers university of technology in Sweden $[6]^{1}$. PALSAR system parameters are first assessed using the Amazonian forest data sets, under low Faraday rotation conditions. The Freeman-VanZyl calibration technique [7], which symmetrizes the system prior to the estimation of the distortion matrix elements, is adopted for this study. The VanZyl algorithm [8], is reconsidered and enhanced using additional equations for unbiased measurement of antenna distortion matrix parameters when azimuthally symmetric reference targets are of low $\mathrm{HV}$ return in comparison with $\mathrm{HH}, \mathrm{VV}$, and the HH-VV cross-correlation [9]. The enhanced VanZyl method is applied on the Amazonian data sets collected in July 2006, for accurate measurements of antenna cross-talk variations with incidence angle, at zero Faraday rotation conditions. It is shown that the antenna is highly isolated with cross-talk lower than $-35 \mathrm{~dB}$. Low Faraday rotation angles (2-3 degree) have been detected on the Sweden site at day acquisitions, on the DLR and the Ottawa calibration sites. The presence of such a low but still significant Faraday rotation angles induces a significant return at the cross-polarization of the corner reflector (-20-25 dB), which might be miss-interpreted as the actual antenna cross-talks, under the assumption of zero Faraday rotation [4], [10].

\section{Assessment of PALSAR SYSTEM PARAMETERS USING THE AMAZONIAN DATA}

Two data sets collected over the Amazonian forest in July 2006 (Ascending mode on the $20^{\text {th }}$ of July , and descending mode on the $21^{\text {st }}$ of July) are used. Corner reflectors (CR) were deployed during the acquisitions for calibration purposes. Faraday rotation is generally low at the Amazonian forests [11], [12]. As a result, conventional Cband calibration methods that are mainly based on the VanZyl algorithm [8] can be used. The response of CR deployed during the acquisitions should permit an accurate measurement of antenna isolation ( $\mathrm{H}-\mathrm{V}$ cross talk).

During the JAXA preliminary calibration process, an-

${ }^{1}$ DLR (K. Papathanassiou) and G. Sandberg, LEB. Eriksson and L. Ulander from Chalmers University of technology are thanked for having kindly provided us with the data and CR information 
tenna gain variations with incidence angle, as well as slant range variations are corrected for [13]. These data will be referred to as "uncalibrated" data. Antenna distortions matrices as well as channel imbalances are then corrected for using antenna distortion matrices measurements obtained from the JAXA site of calibration. This data will be named the "calibrated data".

\section{A. Antenna cross talk measurements using uncalibrated data}

The CR responses in the various polarizations are analyzed using the July 20 (ascending mode) and the July 21 (descending mode) data sets. The CR response in azimuth for the $\mathrm{HH}$ and $\mathrm{VV}$ ascending acquisitions of the 20 of July. $\mathrm{HH}$ and VV response are well focused, with a $3 \mathrm{~dB}$ beamwidth of 1.38 sample in azimuth and 1.12 sample in range. The return in $\mathrm{HV}$ and $\mathrm{VH}$ at the peak location is within the clutter level with a peak to mean clutter ratio of $-0.81 \mathrm{~dB}$ for $\mathrm{HV}$ of Figure 1 . Similar results are obtained with the July 21 descending mode acquisition; the $\mathrm{CR}$ return is within the clutter level and no CR return was noted at the peak location. This leads to the following conclusions:

1. The Faraday rotation is negligible at the Amazonian forest

2. The antenna is highly isolated with a cross-talk lower than the clutter level

At the CR location, the cross-talk is better than $-37 \mathrm{~dB}$ as discussed in Section III.

\section{B. Antenna cross talk measurements using calibrated data}

In the first phase of the calibration phase, the distortion matrix extracted from the Tomakomai site [14], [13] was used to provide calibrated data. Moriyama et al. [14] applied the Quegan method on the Tomakomai site to derive a measure of the PALSAR system transmitting and receiving distortion matrices with cross-talks between -33 $\mathrm{dB}$ and $-28.6 \mathrm{~dB}$. During the first part of the calibration commissioning phase, calibrated data are provided using the Tomakomai distortion matrix [15]. Figure 2 present the $\mathrm{CR}$ response at the $\mathrm{HV}$ polarization for the ascending acquisition of the 20 of July. As can be seen, HV presents a significant return at $\mathrm{HV}$ with a peak to clutter ratio of 13.90 $\mathrm{dB}$ (much higher than the $-0.88 \mathrm{~dB}$ of the uncalibrated image of Figure 1). The distortion matrix that includes low but still significant cross talk induces an artificial and significant cross talk in the "calibrated" data that might be interpreted as the actual antenna cross talk [10], as first brought out in [5].

\section{ANTENNA DISTORTION MATRIX CALIBRATION USING THE AMAZONiAn DATA}

The Amazonian uncalibrated data are used to determine the system distortion matrix [5]. The SIR-C calibration method is adapted to the ALOS data set, and the VanZyl algorithm is enhanced for unbiased estimation of the distortion matrix variations with incidence angle. Antenna crosstalks better than $-35 \mathrm{~dB}$ are recorded along the swath. At the CR location, the cross-talks are better than $-38 \mathrm{~dB}$, and this is in agreement with Moriyama's independent measurements (about $-37 \mathrm{~dB}$ ) based on the Quegan method [16].

Since the Cal-Val meeting (CVS 05), JAXA has provided "calibrated data" using the Amazonian distortion matrix with cross-talks lower than $-37 \mathrm{~dB}[16]$. In fact such low cross-talk may be ignored for the following reasons:

- The cross-talk varies with incidence angles. The measurements we obtained using the enhanced VanZyl algorithm indicates variations of the cross talks along the range swath between $-44 \mathrm{~dB}$ and $-35 \mathrm{~dB}$.

- Measurement of such low cross talks might be affected by the noise floor, and it is safer to set the cross talks to zero, and use diagonal distortion matrix for a more accurate calibration.

In summary, low PALSAR antenna cross talks can be ignored, and diagonal distortions matrix (with zero cross talk) should be used for accurate calibration of PALSAR polarimetric data. This solution is also very convenient in practice since the preliminary JAXA calibration process requires only the correction for the $\mathrm{HH}$ and $\mathrm{VV}$ antenna gains variations, slant range variations, and channel imbalances.

\section{CR RETURN AT THE PRESENCE OF LOW BUt STILL SIGNIFICANT FARADAY ROTATION}

The eventual presence of Faraday rotation in addition to the uncertainty regarding the actual isolation of the $\mathrm{H}$ V PALSAR antenna, leads at the ALOS Cal-Val meeting CVS06 (Tsukuba, March 2007) to mixed conclusions regarding the actual isolation of the H-V PALSAR antenna [17], [18]. At the end of the Cal-Val meeting, the group recommended to provide to all the Cal-Val members the two following data sets for deep analysis:

1. Sweden site of Chalmers university of technology: in this site, L. Eriksson, G. Sandberg, and L. Ulander have deployed $5 \mathrm{~m}$ CR during the ALOS acquisitions [6]. The CRs of high RCS should permit an accurate antenna parameters estimates. The preliminary analysis of data sets collected during day and night [19] reveals significant CR return at the cross-polarization ( $\mathrm{HV}$ and $\mathrm{VH}$ ) during the day acquisitions, and negligible return at night acquisitions.

2. DLR site: The presence of significant CR return at the cross- polarizations leads the authors to the conclusion that the antenna might is badly isolated with a cross talk that can be as low as $-18 \mathrm{~dB}[17],[10]$

In the following, we introduce a simple method that should permits validating the hypothesis of low antenna cross- talks at the presence of low but still significant Faraday rotation. This method is applied on the "calibrated" data provided by JAXA. During, the JAXA calibration process, the distortion matrices and channel imbalance are corrected for using the Amazonian data sets [16]. JAXA measurements reveal very stable channel imbalance ratio [16]. Our measurements confirm the same results with channel imbalance ratio of $\left|f_{2} / f_{1}\right|$ of 0.7 and 0.72 for the 20 th and the 21st of July on the Amazonian site, $\left(\left|f_{2} / f_{1}\right|=0.71\right.$ for the Tomakomai May 2006 acquisition, and $\left|f_{2} / f_{1}\right|=0.712$ for the Ottawa September 2006 
acquisition. As a result, the correction of antenna distortion matrix and channel imbalance measured at the Amazonian sites provide data which only need to be calibrated for the Faraday rotation angle and the transmitter channel imbalance, as follows:

$$
\left[\begin{array}{c}
M_{h h} \\
M_{h v} \\
M_{v h} \\
M_{v v}
\end{array}\right]=\left[\begin{array}{c}
S_{h h} \cos ^{2} \Omega-S_{v v} \sin ^{2} \Omega \\
f_{1}\left[S_{h v}+\left(S_{h h}+S_{v v}\right) \cos \Omega \sin \Omega\right] \\
f_{1}\left[S_{h v}-\left(S_{h h}+S_{v v}\right) \cos \Omega \sin \Omega\right] \\
f_{1}^{2}\left[S_{h h} \cos ^{2} \Omega-S_{v v} \sin ^{2} \Omega\right]
\end{array}\right]
$$

For reciprocal targets, the addition of $\mathrm{HV}$ and VH components eliminates the Faraday rotation angle term, as seen in the equation above. This method is applied on the Sweden (day acquisition) and the DLR data sets that demonstrate significant CR cross-polarization return. Figures 3 and 4 present the $\mathrm{HV}$, and the $\mathrm{HV}+\mathrm{VH} \mathrm{CR}$ responses for the Sweden day acquisitions. As can be noted, whereas the CR shows a significant peak at the HV polarization (with a peak to clutter ratio of about $19 \mathrm{~dB}$, no $\mathrm{CR}$ return is noted at the $\mathrm{HV}+\mathrm{VH}$ with a peak to clutter ratio of about $-8 \mathrm{~dB}$. Similar results are obtained using the Gitching CR of the DLR site. The significant peak at the CR HV polarization (with a peak to clutter ratio of 17.90) vanishes in the $\mathrm{HV}+\mathrm{VH}$ image. These two examples show that the CR return at the cross-pol is not due to antenna crosstalk, as concluded in [17], but rather to the presence of low but still significant Faraday rotation. We run our calibration method on the Sweden and the DLR data sets. This leads to an angle of $\Omega=2.17$ for the Sweden site, which is in agreement with the results obtained in [6]. An angle of $\Omega=2.71$ was found for the DLR site. Such a low angle does not affect significantly the radiometry (within $0.3 \mathrm{~dB}$ ), and as a result, the tests based on the radiometry, such as the histogram of the ratio $\mathrm{HV} / \mathrm{VH}$ derived in [17], cannot reveal the presence of this low but still significant Faraday rotation. In [17], [10], the authors concluded that the Faraday rotation is zero and miss-interpreted the CR return at the cross-polarization as the actual antenna cross-talk. The results above obtained with the sum $\mathrm{HV}+\mathrm{VH}$ prove that the $\mathrm{CR}$ cross-polarization return is rather due to the presence of the low but still significant Faraday rotation effect.

\section{CONClusion}

ALOS PALSAR antenna isolation has been the subject of mixed conclusions. The presence of low but still significant Faraday rotation angle (2-3 degree) induces a significant corner reflector return at the cross-polarization (HV and $\mathrm{VH}$ ) which has been mis-interpreted as the actual antenna cross talk. The Amazonian acquisitions, at free Faraday rotation conditions, permit demonstrating that the PALSAR antenna is highly isolated (better than $-37 \mathrm{~dB}$ at the corner reflector). The enhanced Van Zyl method we developed, which provides accurate antenna distortion parameter measurements, even for targets of low $\mathrm{HV}$ return (with respect to $\mathrm{HH}, \mathrm{VV}$, and the HH-VV crosscorrelation), reveals a cross talk better than $-35 \mathrm{~dB}$ across the swath for both the ascending and descending acquisition over the Amazonian forest. These results are confirmed using the night data acquisition (October 19, 2006) in Sweden, and the September acquisition at low Faraday rotation conditions in Ottawa. They are also in agreement with [20] who have measured cross talk below $-34.5 \mathrm{~dB}$ with the night data acquisition of October 2006. At the presence of significant Faraday rotation, the sum of the calibrated $\mathrm{HV}$ and $\mathrm{VH}$ component cancels the peak at the corner reflector cross-polarization return, as was demonstrated using the data collected over the Sweden and the DLR calibration sites. This confirms the fact that the corner reflector return at $\mathrm{HV}$ and $\mathrm{VH}$ polarization is rather due to the presence of low but still significant Faraday rotation effect. The PALSAR antenna is highly isolated, and a diagonal distortions matrix (with zero cross talk terms) can be used for more accurate calibration [21].

\section{ACKNOWLEDGMENTS}

The authors would like to thank Dr. R.K. Hawkins from CCRS for the helpful discussions and for having organized the calibration campaign, K. Murnagham (CCRS) for helping deploy the corner reflector, and S. Nedelcu (CCRS) for his help in PALSAR data processing.

\section{REFERENCES}

[1] N. Ito, T. Hamazaki, and K. Tomioka. ALOS/PALSAR chracteristics and status. In CEOS SAR Workshop Proc., Tokyo, pages 191-194, April 2001.

[2] M. Shimada. Calibration and validation of PALSAR and research products of NASDA/EORC. In Proc. of CEOS'01 Workshop, Tokyo, Japan, 2001.

[3] E. Rignot. Effect of Faraday rotation on L-band interferometric and polarimetric Synthetic Aperture Radar data. IEEE Trans. Geoscience Rem. Sens., 38(1):383-390, 2000.

[4] K.P. Papathanassiou and R. Zandona-Schneider. ALOS PALSAR- PolInSAR data analysis: First results. In Presented at the ALOS Cal-Val meeting CVST5, Tokyo, Japan, Oct. 2006.

[5] R. Touzi, M. Shimada, S. Nedelcu, R.K. Hawkins, and K. Murnagham. Calibration of PALSAR polarimetric modes: SAR system model assessment using the Amazonian and Ottawa calibration site data. In ALOS Cal-Val meeting CVST5, Tokyo, Japan, Oct. 2006.

[6] G.Sandberg, L.E.B. Eriksson, and L.M.H. Ulander Faraday rotation measured in polarimetric PALSAR images compared with theoretical expectations. In Proc. of ASAR 2007, Vancouver, Canada, Sep. 2007.

[7] A. Freeman, J.J. Van Zyl, J.D. Klein, H.A. Zebker, and Y. Shen. Calibration of Stokes and scattering matrix format polarimetric SAR data . IEEE Trans. Geoscience Rem. Sens., 30(3):531-539, 1992

[8] J.J. Van Zyl. Calibration of polarimetric radar images using only image parameters and trihedral corner reectors responses. IEEE Trans. Geoscience Rem. Sens., 28(3):337-348, 1990.

[9] R. Touzi, C. E. Livingstone, J. R. C. Lafontaine, and T. I. Lukowski. Consideration of antenna gain and phase patterns for calibration of polarimetric SAR data. IEEE Trans. Geoscience Rem. Sens., 31(6):1132-1145, 1993.

[10] K.P. Papathanassiou. ALOS PALSAR- PolInSAR data analysis: First results. In Presented at the Pol-InSAR 200\%, Frascatti, Italy, Jan. 2007.

[11] P. Wright, S. Quegan, N. Wheadon, and D. Hall. Faraday rotation effects on L-band spaceborne SAR data. IEEE Trans. Geoscience Rem. Sens., 41:2735-2744, 2003.

[12] M. Shimada. Long-term stability of L-band normalized RCS of Amazon rain forest using JERS-1 SAR. In Proc. of ASAR/CEOS 2003, Montreal, Canada, 24-27 June 2003. 
[13] M. Shimada, T. Tadono, M. Watanabe, and A. Resenqvist. Calibration and validation of PALSAR (version 5). In ALOS Cal-Val meeting CVST4, Tokyo , Japan, June 2006.

[14] T. Moriyama. PALSAR polarimetric evaluation. In Presented at the ALOS Cal-Val meeting CVST5, Tokyo, Japan, Oct. 2006.

[15] M. Shimada. PALSAR CAL VAL Report to CVST5. In ALOS Cal-Val meeting CVST5, Tokyo , Japan, Oct. 2006.

[16] M. Shimada. PALSAR CAL VAL Report to CVST6. In ALOS Cal-Val meeting CVST6, Tsukuba, Japan, March 2007.

[17] K.P. Papathanassiou and R. Zandona-Schneider. ALOS PALSAR- PolInSAR data analysis: First results. In Presented at the ALOS Cal-Val meeting CVST6, Tsukuba, Japan, March 2007.

[18] R. Touzi, M. Shimada, S. Nedelcu, R.K. Hawkins, and K. Murnagham. PALSAR polarimetric calibration and Ottawa data quality assessment. In ALOS Cal-Val meeting CVST6, Tsukuba, Japan, March 2007.

[19] L. Ulander, J. Fransson, and L. Eriksson. PALSAR Cal/Val results from Sweeden. In ALOS Cal-Val meeting CVST5, Tokyo , Japan, Oct. 2006.

[20] G.Sandberg, L.E.B. Eriksson, and L.M.H. Ulander. Measurement of Farady rotation using polarimetric PALSAR images. IEEE Geoscience and Rem. Sens. Letters, 6(1), January 2009.

[21] R. Touzi, , and M. Shimada. polarimetric PALSAR Calibration. IEEE Trans. Geoscience Rem. Sens., Vol. 47, No. 12:3951-3959, Special issue on ALOS, December, 2009.

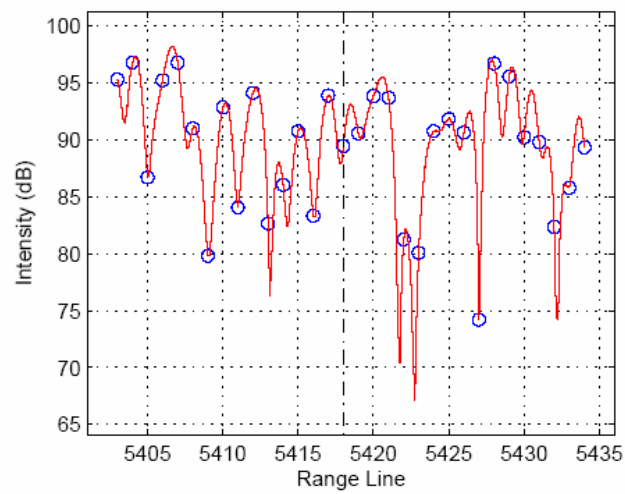

Fig. 1. Uncalibrated HV Corner Reflector azimuth response

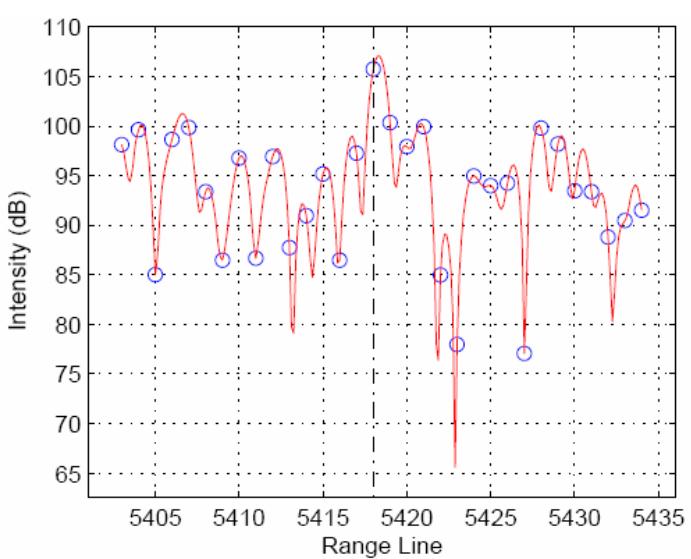

Fig. 2. Calibrated HV Corner Reflector azimuth response

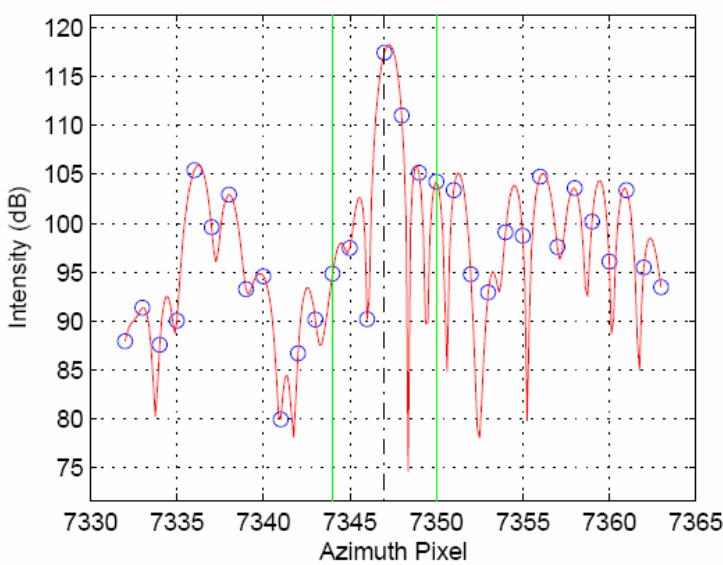

Fig. 3. Calibrated Sweden HV Corner Reflector azimuth response

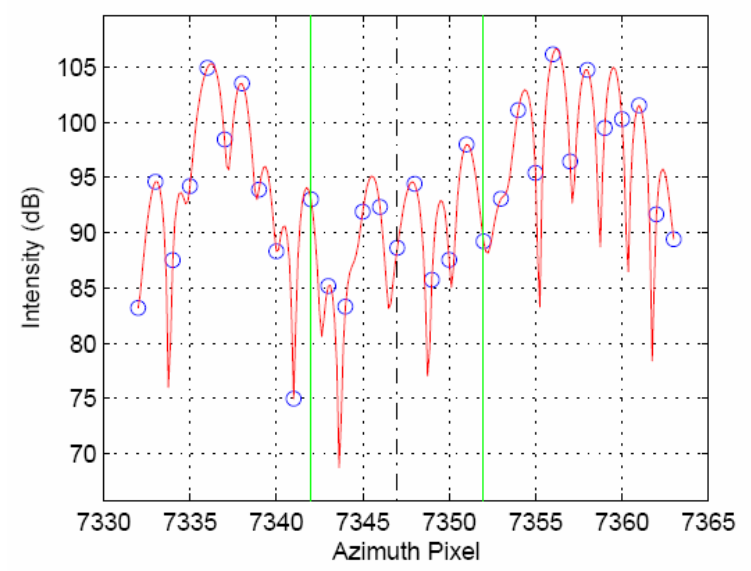

Fig. 4. $|H V+V H|$ Corner Reflector azimuth response 